\title{
NLRP3 inflammasome: a novel link between lipoproteins and atherosclerosis
}

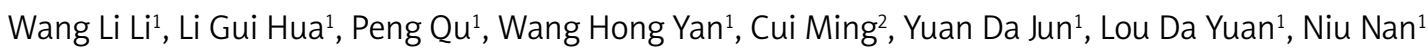

\author{
${ }^{1}$ Department of Cardiology, Second Affiliated Hospital of Dalian Medical University, \\ Dalian, Liaoning, China \\ ${ }^{2}$ Department of Emergency, Second Affiliated Hospital of Dalian Medical University, \\ Dalian, Liaoning, China
}

Submitted: 29 October 2014

Accepted: 30 December 2014

Arch Med Sci 2016; 12, 5: 950-958

DOI: 10.5114/aoms.2016.61356

Copyright @ 2016 Termedia \& Banach

\section{Abstract}

Introduction: Pattern recognition receptor-mediated signaling pathways have recently been elucidated to bridge the innate immune system and atherosclerosis. NLRP3 is a member of the NLR family. Upon activation, it initiates IL-1 $\beta$ and IL-18 processing, a key step in the inflammatory process of atherosclerosis.

Material and methods: We used three different types of lipoproteins, ox$\mathrm{LDL}$, ox-HDL, and HDL, in Thp-1 at the concentration of $50 \mathrm{mg} / \mathrm{l}, 100 \mathrm{mg} / \mathrm{l}$, and $150 \mathrm{mg} / \mathrm{l}$ respectively. Using real-time polymerase chain reaction and western blot, ELISA detected the expression of NLRP3 and downstream cytokines. NLRP3 siRNA was constructed to down-regulate expression of the NLRP3 gene via the RNA interference technique. $150 \mathrm{mg} / \mathrm{l}$ of ox-LDL, ox-HDL and HDL was added to the Thp-1 cell line respectively. We observed the changes in the expression of caspase-1, IL-1 $\beta$ and IL-18 when the NLRP3 gene was down-regulated.

Results: Ox-LDL and ox-HDL addition not only increases the expression of NLRP3, but also activates the NLRP3 downstream cytokines and caspase- 1 and induces IL-1 $\beta$ and IL-18 secretion. Moreover, the effects of activation and induction are shown to have a dose-dependent manner. Expression of NLRP3 and its downstream inflammatory cytokines is reduced in the presence of HDL $(p<0.05)$. Furthermore, our data demonstrated that NLRP3 siRNA downregulates NLRP3 expression in mononuclear cells, thus leading to a dramatic reduction in the expression of caspase- $1, \mathrm{IL}-1 \beta$ and IL-18 $(p<0.05)$.

Conclusions: The data suggest that activation of the NLRP3 inflammasome is a critical step in caspase- 1 activation and IL- $1 \beta$ and IL-18 secretion. Interference with the NLRP3 inflammasome can significantly inhibit the generation of cytokines, thus impeding the pathogenesis of inflammation.

Key words: NLRP3, inflammasome, atherosclerosis, monocytes, lipoprotein.

\section{Introduction}

Chronic inflammation is an important mechanism in the progression of atherosclerosis. Lipid deposition on the arterial wall and infiltration of inflammatory cells are considered as the main events in the process of atherosclerosis [1]. Abnormal lipoprotein metabolism is considered as an important risk factor in the pathogenesis of atherosclerosis. The abnormal lipoprotein metabolism involvement in the pathogenesis and progression of atherosclerosis has multiple complicated mechanisms.

\author{
Corresponding author: \\ Dr. Peng Qu \\ Department of Cardiology \\ Second Affiliated Hospital \\ of Dalian Medical University \\ Zhong shan Road No. 467 \\ Dalian \\ 116023 Liaoning, China \\ Phone: +86-411846712913021 \\ Fax: +86-411846712913021 \\ E-mail: Pengqu_In@hotmail. \\ com
}


A large number of recent studies have suggested that pattern-recognition receptors (PRRs)-mediated inflammation plays a key role in the process of atherosclerosis by abnormal lipoprotein, of which TLR2, TLR4 and LOX-1 have been studied most [2-4]. At present, researchers have turned their attention to the study of NLRP3 inflammasome, a member of the NLR family. Our study found that NLRP3 and downstream cytokines were coupled with increasing severity of coronary artery disease, and dynamic variation exists in patients with acute myocardial infarction [5]. Duewell et al. [6] reported that cholesterol crystal is able to activate the NLRP3 inflammasome in macrophages during early formation of atherosclerosis. Intraperitoneal injection of cholesterol crystals in mice can induce acute inflammation. However, this induction is dramatically reduced in mice defective in the NLRP3 inflammasome. Bone marrow transplantation of NLRP3, ASC or IL-1 knock-out mice followed by a high-lipid diet can significantly reduce the early formation of atherosclerosis and inflammation. These data suggest that the NLRP3 inflammasome mediated signaling regulates cholesterol crystal induced inflammation in atherosclerosis.

However, the mechanism whereby the NLRP3 inflammasome mediates the abnormal lipoprotein metabolism in chronic inflammation of atherosclerosis has not been fully clarified. In addition, the effect of lipoproteins on the NLRP3 inflammasome also remains unclear. In this study, we addressed the effect of three different lipoproteins - ox-LDL, ox-HDL and HDL - on the expression of NLRP3 and its downstream cytokines in a human mononuclear cell line (Thp-1). In addition, we further explored the possible mechanism of ox-LDL, oxHDL and HDL-mediated effects on NLRP3 leading to atherosclerotic inflammation. We also explored the potential of NLRP3 to be used as a therapeutic target in atherosclerosis.

\section{Material and methods}

\section{Cell culture}

Human mononuclear cell line Thp-1 was purchased from Cell Resource Center of Shanghai Life Science Institute, Chinese Academy of Science. Cell lines were incubated in PRIM1640 containing $10 \%$ fetal bovine serum with a humidified atmosphere of $95 \%$ air and $5 \% \mathrm{CO}_{2}$ at $37^{\circ} \mathrm{C}$. Cells were incubated in a 6 -well culture dish with $80-90 \%$ confluency to begin the next experiment. In this study, three different types of lipoproteins - ox-LDL, ox-HDL and HDL - were added to the cell culture medium at the concentrations of $50 \mathrm{mg} / \mathrm{l}, 100 \mathrm{mg} / \mathrm{l}$ and $150 \mathrm{mg} / \mathrm{l}$ respectively. The cells and supernatant were harvested for the subsequent experiments after $24 \mathrm{~h}$. The ox-LDL, ox-HDL and HDL were from Beijing Pulizhicheng Biological Technology Co. Ltd. The oxidation was performed with $\mathrm{Cu}^{2+}$ and the Lipid Peroxidation MDA Assay Kit was used to make sure of a similar degree of oxidation for the lipoproteins, and then diluted to the required concentration with cell culture medium.

\section{Synthetic siRNAs and transient transfection}

NLRP3 siRNA was constructed to down-regulate expression of the NLRP3 gene via the RNA interference technique. We observed the change in the expression of caspase-1, IL-1 $\beta$ and IL-18 when the NLRP3 gene was down-regulated. NLRP3 siRNA was designed and synthesized by GuangZhou Ruibo Biological Technology Co. Ltd. The target sequence of the NLRP3 siRNA was GAAGTGGATTGAAGTGAAA, the sense strand was (5'-3') GAAAUGGAUUGAAGUGAAAdTdT and the antisense strand was (3'-5') dTdTCUUUACCUAACUUCACUUU. The cells were incubated in a 6-well culture dish with $60-80 \%$ confluency to begin transfection. The transient transfection reagent was Lipofectamine 2000 (Invitrogen, Carlsbad, CA). The NLRP3 siRNAs were mixed with Lipofectamine 2000 according to the recommendations in the manual. The transfection solution was then added to a 6-well culture dish after 20 min standing, and replaced to new culture medium after $6 \mathrm{~h}$. Then ox-LDL, ox-HDL and HDL were added to the cell culture medium at the concentration of $150 \mathrm{mg} / \mathrm{l}$. The cells and supernatant were harvested for the subsequent experiment after $24 \mathrm{~h}$.

\section{Western blot analysis}

Western blot was used to examine the expression levels of NLRP3, caspase- $1, \mathrm{IL}-1 \beta, \mathrm{IL}-18$ and GAPDH. Cells were washed with cold PBS, and total proteins were then extracted from cells using a total protein extraction kit (KeyGEN, Nanjing, China) according to the recommendations in the manual. After protein quantification using a BCA protein assay kit (KeyGEN, Nanjing, China), 80-100 $\mu \mathrm{g}$ of the proteins were heated to $95^{\circ} \mathrm{C}$ for $5 \mathrm{~min}$. Denatured proteins were separated using $12 \%$ SDS-PAGE and were then transferred to the nitrocellulose membrane. The membrane was blocked using a confining liquid (5\% nonfat milk powder in TTBS) at $4^{\circ} \mathrm{C}$ overnight. The antibodies were incubated with the most suitable concentration for $4 \mathrm{~h}$ and were then washed separately using TTBS. The HPR-mer antibodies were incubated for $1 \mathrm{~h}$ and then washed. Results were detected by ECL Plus western blot detection reagents (KeyGEN, Nanjing, China). The amount of protein was determined by densitometry using LabWorks software (UVP, Up- 
land, CA). The antibody for NLRP3 was from Abcam (ab17267), antibody for pro-caspase-1 and caspase-1 were from Sigma-Aldrich (C4851), antibody for pro-IL-1 $\beta$ (MAB6964), IL-1 $\beta$ (MAB201) and pro-IL-18 (MAB 646) were from R\&D Systems, and antibody for IL-18 was from MBL (D043-3).

\section{Real-time fluorescent quantitative PCR}

Total RNA was isolated using the Trizol reagent (Sigma) method. The yield of RNA was subjected to reverse transcription using the MMLV reverse transcription kit (Promega). Then, cDNA was subjected to qPCR for NLRP3, caspase-1, IL-1 $\beta$, IL-18 and the $\beta$-actin gene using the SYBR Green PCR kit (Promega). Quantification was done by normalization against $\beta$-actin. The following primer sequences were used: $\beta$-actin upstream primer: 5'-CGTGACATTAAGGAGAAGCTG-3', downstream primer: 5'-CTAGAAGCATTTGCGGTGGAC-3'; NLRP3 upstream primer: 5'-CTTCCTTTCCAGTTTGCTGC-3', downstream primer: 5'-TCTCGCAGTCCACTTCCTTT-3'; caspase-1 upstream primer: 5'-GCCCAAGTTTGAAGGACAAA-3', downstream primer: 5'-GGTGTGGAAGAGCAGAAAGC-3'; IL-1 $\beta$ upstream primer: 5'-GCCCTAAACAGATGAAGTGCTC-3', downstream primer: 5'-GAACCAGCATCTTCCTCAG-3'; IL-18 upstream primer: 5'-GCTTGAATCTAAATTATCAGTC-3', downstream primer: 5'-GAAGATTCAAATTGCATCTTAT-3'.

\section{Cytokine measurement}

$\mathrm{IL}-1 \beta$ and IL-18 concentrations were measured in the supernatants of each cell culture using the human IL-1 $\beta$ and IL-18 Platinum ELISA kits (eBioscience, USA).

\section{Statistical analysis}

All data were analyzed using SPSS Statistics 13.0, and each analysis provided at least three results. The values are expressed in mean \pm SD . Mean comparison between two groups and among multiple groups $(\geq 3)$ was analyzed by the $t$-test and one-way ANOVA respectively. Percentage comparison among multiple groups $(\geq 3)$ was analyzed by the $\chi^{2}$ test. $P$-value $<0.05$ was considered to be statistically significant.

\section{Results}

Ox-LDL, ox-HDL and HDL induced expression of NLRP3 and its downstream cytokines in human mononuclear cells

In this study, we found that the mRNA level of NLRP3 is dramatically increased in the experimental groups of ox-LDL and ox-HDL as compared to the control group (in the absence of ox-LDL and ox$\mathrm{HDL}$ ). Moreover, the increase in the mRNA levels is ox-LDL and ox-HDL concentration dependent. In contrast, in the presence of HDL, the mRNA level of NLRP3 is decreased, and this inhibitory effect is also dependent on the concentration of HDL. We also found that the mRNA level of NLRP3 downstream cytokines including caspase-1, IL-1 $\beta$ and IL-18 has a similar pattern to the level of NLRP3. In the presence of ox-LDL and ox-HDL, the mRNA levels of caspase-1, IL-1 $\beta$ and IL-18 are increased as compared to the control, and this increase is ox-LDL and ox-HDL concentration dependent. However, in the presence of HDL, the mRNA levels of these three cytokines are significantly reduced. The reduction in the mRNA levels of caspase-1, $\mathrm{IL}-1 \beta$ and IL-18 is also concentration dependent and is statistically significant as compared to the control group (Figure 1).

To better understand the potential role of NLRP3 in atherosclerotic inflammation, we also determined the expression of NLRP3 and its downstream inflammatory cytokines as they function as a multi-protein complex. The data clearly indicated that the expression of NLRP3 is enhanced in ox-LDL and ox-HDL groups, and the concentration dependence is more pronounced in the oxLDL group. However, the level of NLRP3 expression is only increased at the high concentration of ox-HDL (100 mg/l and $150 \mathrm{mg} / \mathrm{l})$ as compared to the control, and there is no significant difference in the expression of NLRP3 between these two concentrations. In contrast, the expression of NLRP3 is diminished in the presence of HDL in a concentration-dependent manner. NLRP3-induced activation of caspase- 1 is more pronounced in the presence ox-LDL or ox-HDL. In particular, there is a remarkable increase in the expression of caspase- 1 at a high concentrations of ox-LDL (100 mg/l and $150 \mathrm{mg} / \mathrm{l}$ ) as compared to the control. However, there is no statistically significant difference between these two concentrations. In the group of ox-HDL, the caspase- 1 expression can only be induced at the high concentration of $100 \mathrm{mg} / \mathrm{l}$ or $150 \mathrm{mg} / \mathrm{l}$, and no statistically significant difference is observed between these two concentrations. In contrast, the level of caspase- 1 expression is reduced in the presence of HDL, and this inhibitory effect is also concentration dependent. Activation of caspase- 1 can further promote the splicing and modification of its downstream cytokines, IL-1 $\beta$ and IL-18 precursor and induce the maturation and secretion of IL-1 $\beta$ and IL-18. We also determined the expression of IL-1 $\beta$ and IL-18 via Western blotting analysis. It showed that the IL-1 $\beta$ expression is only increased at the concentration of ox-LDL (100 mg/l, $150 \mathrm{mg} / \mathrm{l})$ as compared to the control, but there is a statistically significant difference between these two concentrations. No change in the expression of IL- $1 \beta$ at the concentrations of $50 \mathrm{mg} / \mathrm{l}$ and $100 \mathrm{mg} / \mathrm{l}$ of ox-HDL was observed as compared to the control. 
A

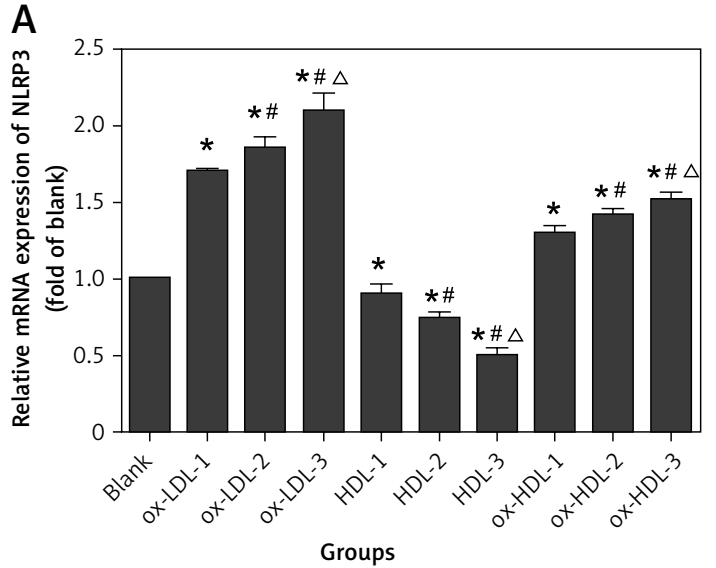

C

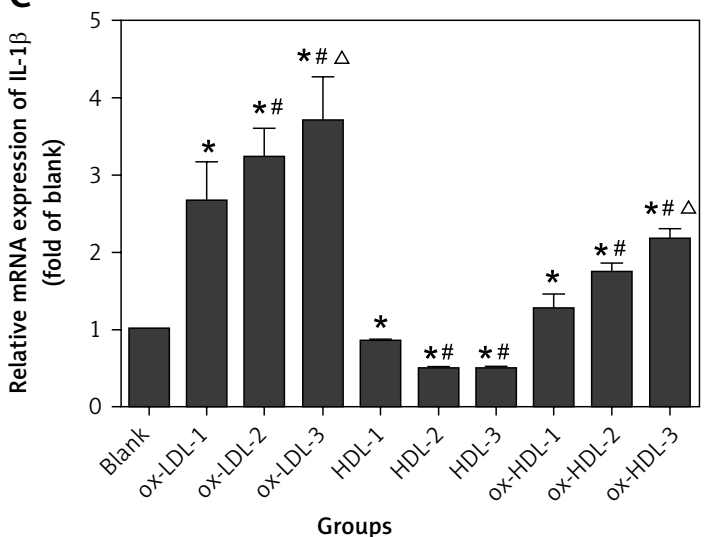

B

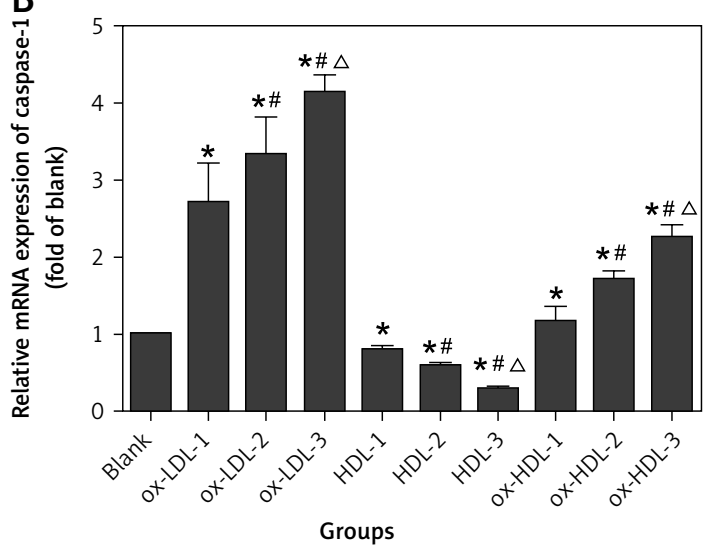

D

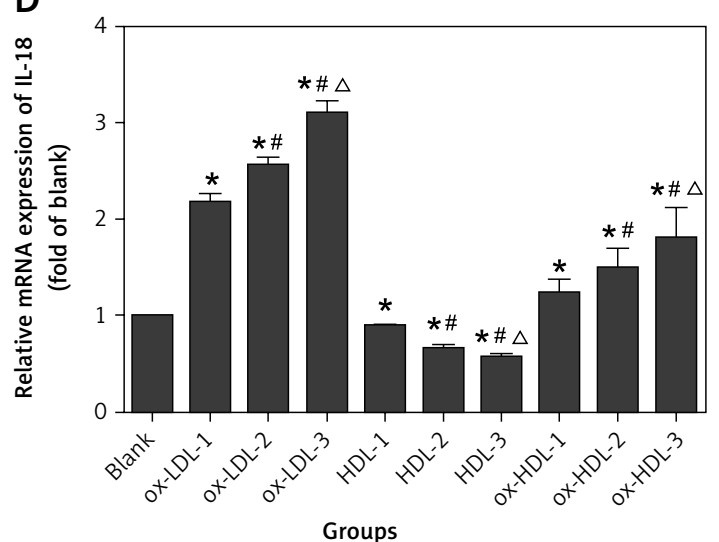

Figure 1. Ox-LDL, ox-HDL and HDL induced mRNA expression of NLRP3 and its downstream cytokines in human mononuclear cells

Blank - blank control group, HDL-1 - group of HDL at the concentration of $50 \mathrm{mg} / \mathrm{l}, \mathrm{HDL}-2$ - group of HDL at the concentration of $100 \mathrm{mg} / \mathrm{l}, \mathrm{HDL}-3$ - group of HDL at the concentration of $150 \mathrm{mg} / \mathrm{l}, \mathrm{ox}-L D L 1$ - group of ox-LDL at the concentration of $50 \mathrm{mg} / \mathrm{l}$, ox$L D L-2$ - group of ox- $L D L$ at the concentration of $100 \mathrm{mg} / \mathrm{l}$, ox- $L D L-3$ - group of ox- $L D L$ at the concentration of $150 \mathrm{mg} / \mathrm{l}$, ox-HDL 1 - group of ox-HDL at the concentration of $50 \mathrm{mg} / \mathrm{l}, \mathrm{ox}-\mathrm{HDL}-2$ - group of ox-HDL at the concentration of $100 \mathrm{mg} / \mathrm{l}, \mathrm{ox}-\mathrm{HDL}-3-$ group of ox-HDL at the concentration of $150 \mathrm{mg} / \mathrm{l}$. Each set of $H D L, o x-L D L, o x-H D L$ concentration after processing, ${ }^{*} p<0.05$, compared with the blank control group was statistically significantly different; " $p<0.05$, compared with $50 \mathrm{mg} / \mathrm{l}$ group was statistically significantly different, ${ }^{\Delta} p<0.05$, compared with $100 \mathrm{mg} / \mathrm{l}$ group was statistically significantly different.

However, at the concentration of $150 \mathrm{mg} / \mathrm{l}$, the expression of IL-1 $\beta$ is dramatically enhanced. In contrast, the expression of IL- $1 \beta$ is inhibited in the presence of HDL as compared to the control group. However, no statistically significant difference was observed between the concentrations of $100 \mathrm{mg} / \mathrm{l}$ and $150 \mathrm{mg} / \mathrm{l}$ of $\mathrm{HDL}$. The expression of IL-18 is significantly increased in the presence of ox-LDL although there is no statistically significant difference between the concentration of $50 \mathrm{mg} / \mathrm{l}$ and $100 \mathrm{mg} / \mathrm{l}$. However, the ox-LDL induction of IL-18 is more evident at the concentration of $150 \mathrm{mg} / \mathrm{l}$. The IL-18 expression is remarkably enhanced in an ox-HDL concentration dependent manner. In comparison, HDL addition has a statistically significant inhibitory effect on the expression of IL-18 as compared to the control. However, no statistically significant difference was detected between $100 \mathrm{mg} / \mathrm{l}$ and $150 \mathrm{mg} / \mathrm{l}$ of HDL (Figure 2).

It is well known that IL-1 $\beta$ and IL-18 are required to be secreted to the cytoplasm to have biological functions after they are synthesized in mononuclear cells. In this study, IL-1 $\beta$ and IL-18 concentrations were measured in the supernatants of each cell cultures. Secretion of IL-1 $\beta$ and IL-18 to the cytoplasm is too low to be detected in HDL and control groups. In contrast, ELISA analysis clearly indicated that the expression of IL-1 $\beta$ and $\mathrm{IL}-18$ is dramatically enhanced in the presence of ox-LDL or ox-HDL in a concentration-dependent manner (Figure 3).

The data indicate that the lipoproteins ox-LDL and $\mathrm{Ox}-\mathrm{HDL}$ enhance the expression of NLRP3 and promote the assembly and biological activity of the NLRP3 inflammasome. In addition, they can further activate the downstream effector of the NLRP3 inflammasome, caspase-1, and induce the secretion of IL-1 $\beta$ and IL-18, which are concentration-dependent. However, in the presence of HDL, the expression of NLRP3 and its downstream inflammatory factors is inhibited. This suggests that $\mathrm{HDL}$ plays a protective role in the pathogenesis of 
A

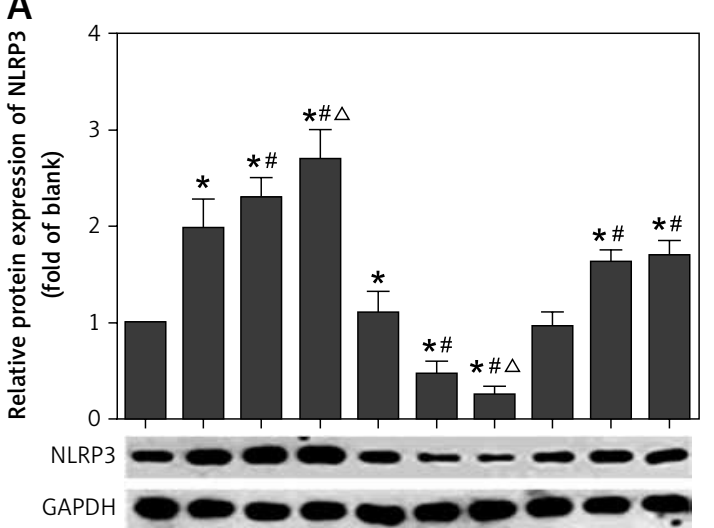

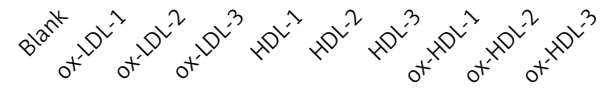

Groups

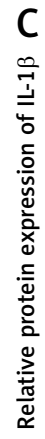

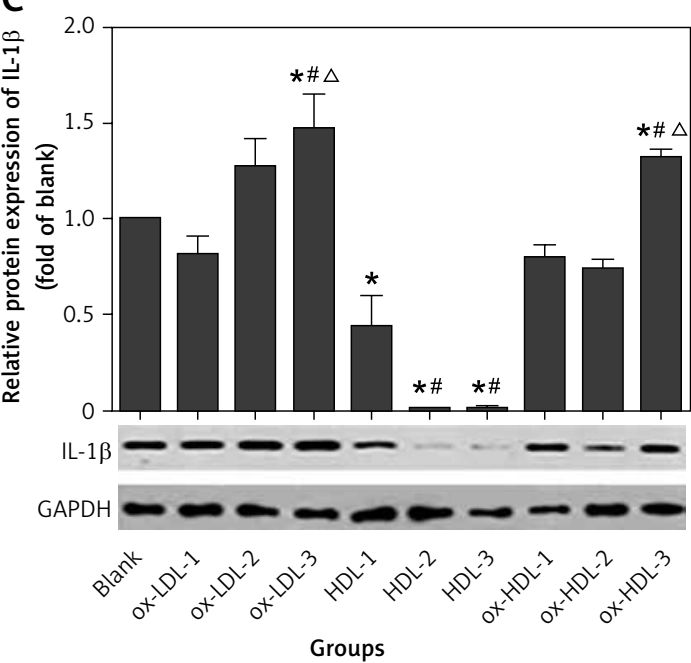

B

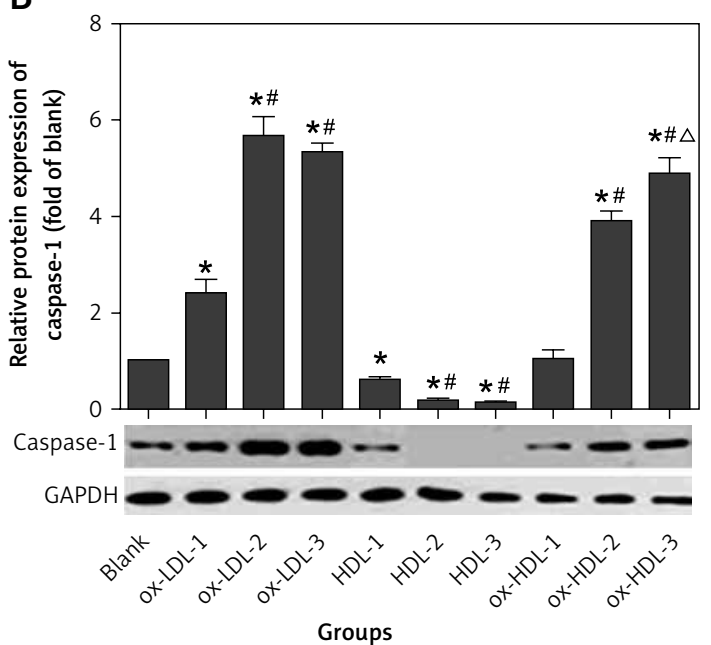

D

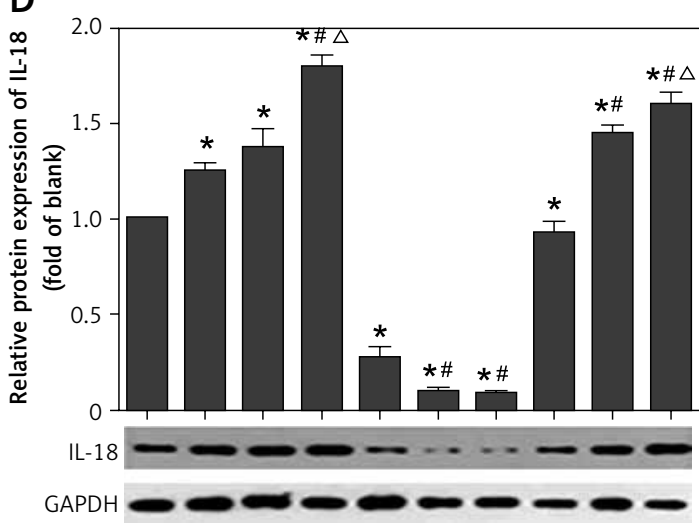

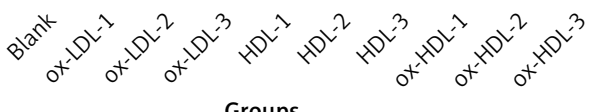

Groups

Figure 2. Ox-LDL, ox-HDL and HDL induced the protein expression of NLRP3 and its downstream cytokines in human mononuclear cells

The sample order of Western blot from left to right: blank, ox-LDL-1, ox-LDL-2, ox-LDL-3, HDL-1, HDL-2, HDL-3, ox-HDL-1, ox$H D L-2$, ox-HDL-3. Blank - blank control group, HDL-1-group of HDL at the concentration of $50 \mathrm{mg} / \mathrm{l}$; HDL-2-group of HDL at the concentration of $100 \mathrm{mg} / \mathrm{l}, \mathrm{HDL}-3$-group of $H D L$ at the concentration of $150 \mathrm{mg} / \mathrm{l}$, ox-LDL-1-group of ox-LDL at the concentration of $50 \mathrm{mg} / \mathrm{l}$, ox- $L D L-2$ - group of ox- $L D L$ at the concentration of $100 \mathrm{mg} / \mathrm{l}$, ox- $L D L-3$ - group of ox-LDL at the concentration of $150 \mathrm{mg} / \mathrm{l}$, ox-HDL-1 - group of ox-HDL at the concentration of $50 \mathrm{mg} / \mathrm{l}$, ox-HDL-2 - group of ox-HDL at the concentration of $100 \mathrm{mg} / \mathrm{l}$, ox-HDL-3 - group of ox-HDL at the concentration of $150 \mathrm{mg} / \mathrm{l}$. Each set of HDL, ox-LDL, ox-HDL concentration after processing, ${ }^{*} p<0.05$, compared with the blank control group was statistically significantly different, ${ }^{*} p<0.05$, compared with $50 \mathrm{mg} / \mathrm{l}$ group was statistically significantly different, ${ }^{\Delta} p<0.05$, compared with $100 \mathrm{mg} / \mathrm{lg}$ group was statistically significantly different.

atherosclerosis by inhibiting NLRP3 and its downstream inflammatory cytokines.

NLRP3 siRNA interferes with the expression of NLRP3 and its downstream effectors including caspase-1, IL-1 $\beta$ and IL-18

We found that ox-LDL, ox-HDL and HDL have biological effects on the expression of NLRP3 and its downstream effectors (caspase-1, IL-1 $\beta$ and IL-18). This suggests that ox-LDL, Ox-HDL and HDL are involved in the pathogenesis of atherosclerosis by affecting the function of the NLRP3 inflammasome. In this experiment, NLRP3 siRNA was constructed to down-regulate expression of the NLRP3 gene via the RNA interference technique. The data showed that expression of NLRP3 is significantly reduced by NLRP3 SiRNA in the groups of ox-LDL, ox-HDL and HDL. On average, the level of reduction in the expression of NLRP3 is $60-70 \%$. However, the inhibition levels among the three groups are not statistically significantly different (Figure 4 A). We also found that the level of caspase- 1 expression is approximately $70 \%$ decreased in each group transfected with NLRP3 siRNA, and the inhibition level among groups is not statistically significantly different. The data clearly indicated that interference with NLRP3 can effectively inhibit the expression of caspase-1 (Figure 4 B). 
A

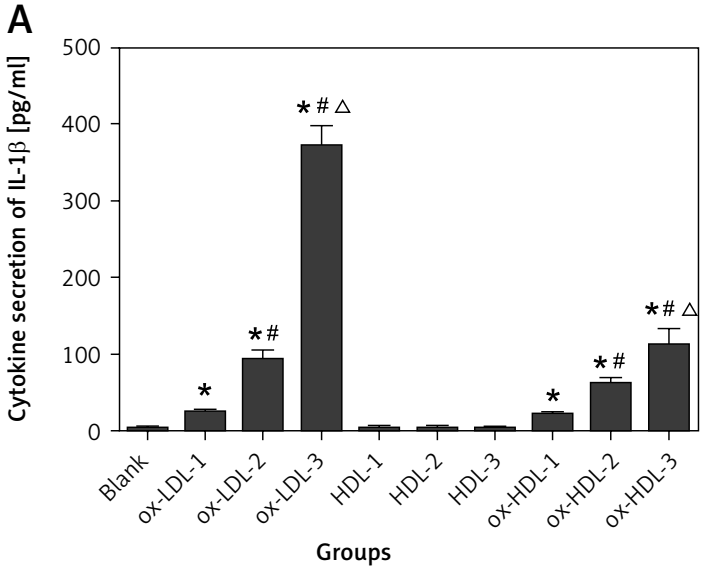

B

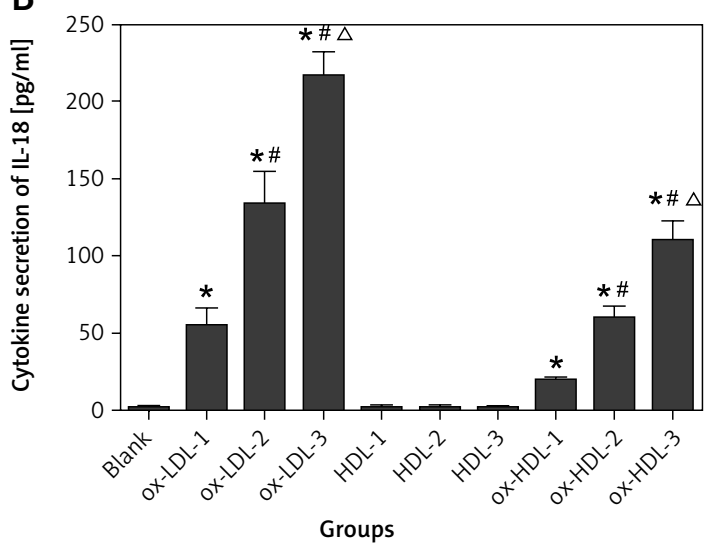

Figure 3. Ox-LDL, ox-HDL and HDL induced secretion of IL-1 $\beta$ and IL-18 in the supernatant of cell culture

Blank - blank control group, HDL-1 - group of HDL at the concentration of $50 \mathrm{mg} / \mathrm{l}, \mathrm{HDL}-2$ - group of HDL at the concentration of $100 \mathrm{mg} / \mathrm{l}, \mathrm{HDL}-3$ - group of $H D L$ at the concentration of $150 \mathrm{mg} / \mathrm{l}, \mathrm{ox}-\mathrm{LDL}-1$ - group of ox-LDL at the concentration of $50 \mathrm{mg} / \mathrm{l}$, ox-LDL-2 - group of ox-LDL at the concentration of $100 \mathrm{mg} / \mathrm{l}$, ox- $L D L-3$ - group of ox-LDL at the concentration of $150 \mathrm{mg} / \mathrm{l}$, ox-HDL-1 - group of ox-HDL at the concentration of $50 \mathrm{mg} / \mathrm{l}$, ox-HDL-2 - group of ox-HDL at the concentration of $100 \mathrm{mg} / \mathrm{l}$, ox-HDL-3 - group of ox-HDL at the concentration of $150 \mathrm{mg} / \mathrm{l}$. Each set of $H D L$, ox- $L D L, o x-H D L$ concentration after processing, ${ }^{*} p<0.05$, compared with the blank control group was statistically significantly different, ${ }^{*} p<0.05$, compared with $50 \mathrm{mg} / \mathrm{lgroup}$ was statistically significantly different, ${ }^{\Delta} p<0.05$, compared with $100 \mathrm{mg} / \mathrm{l}$ group was statistically significantly different.

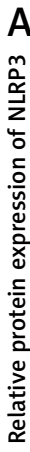

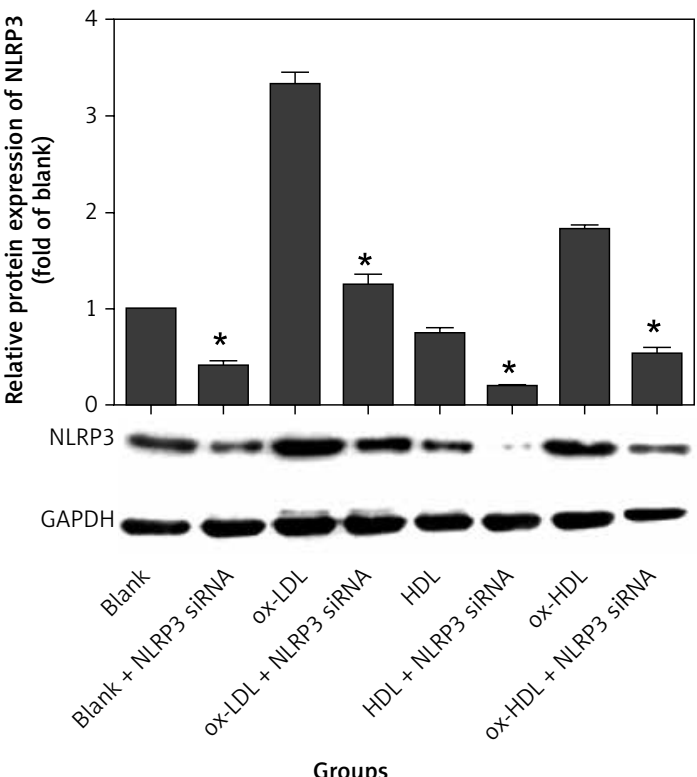

B
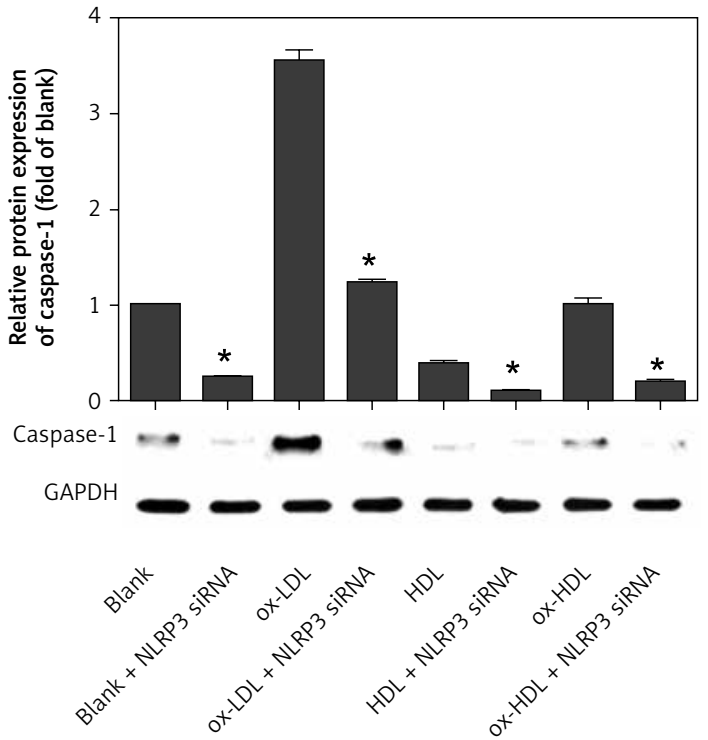

Groups

Figure 4. NLRP3 siRNA interferes with expression of NLRP3 and caspase-1

${ }^{*} p<0.05$, statistically significantly different compared to the group which did not transfect with NLRP3 siRNA.

We further examined the expression of IL-1 $\beta$ and IL-18 in the supernatant of cell culture using ELISA. IL-1 $\beta$ and IL-18 levels were too low to be detected in the groups of control, control transfected with NLRP3 siRNA, HDL and HDL transfected with NLRP3 siRNA. However, the expression of IL-1 $\beta$ and IL-18 is significantly decreased in the groups of ox-LDL and ox-HDL transfected with NLRP3 SiRNA respectively. On average, the reduction level is approximately $60-70 \%$. However, there is no statistically significant difference between the groups of ox-LDL and ox-HDL (Figure 5).
Taken together, our data showed that NLRP3 SiRNA effectively reduces the expression of NLRP3 and its downstream effectors (caspase-1, IL-1 $\beta$ and IL-18). This suggests that downregulation of NLRP3 inhibits the occurrence of the inflammatory reaction by affecting the NLRP3 downstream effectors including caspase-1, IL-1 $\beta$ and IL-18.

\section{Discussion}

Abnormal lipid profiles are usually considered as a vital index of atherosclerotic cardiovascular 
A

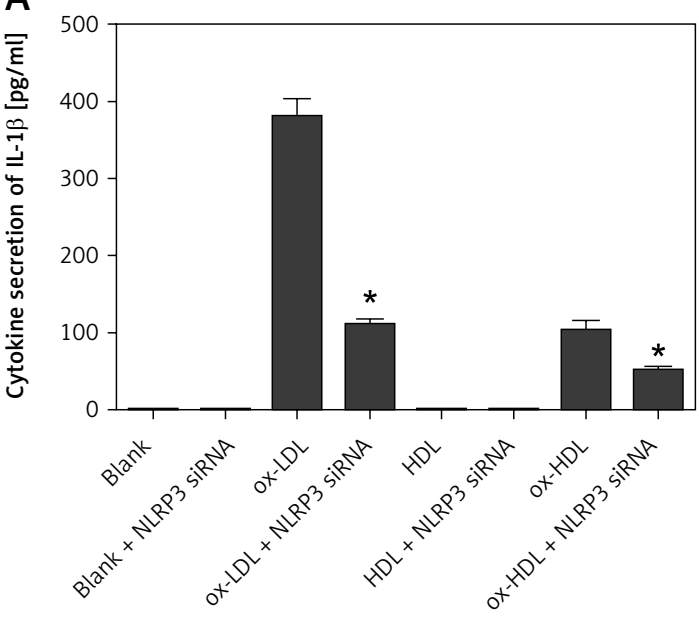

Groups
$B$

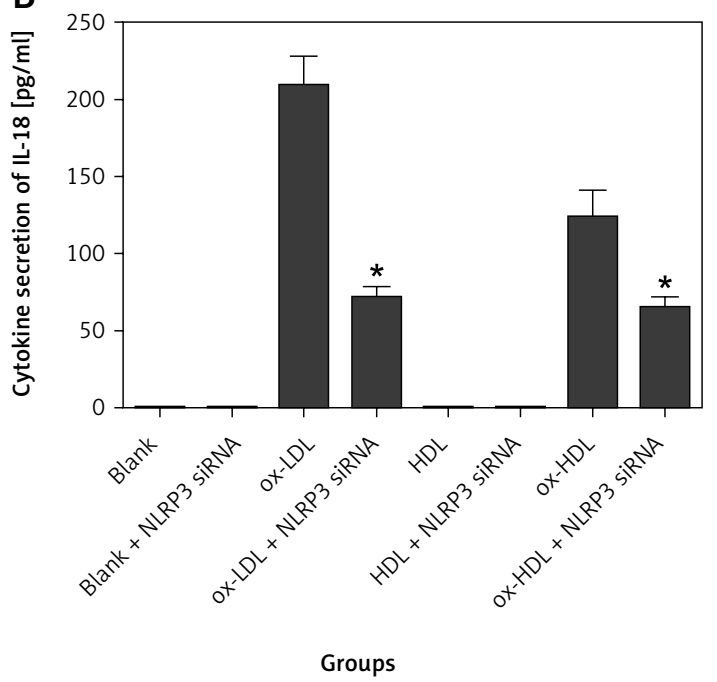

Figure 5. NLRP3 SiRNA interferes with secretion of IL-1 $\beta$ and IL-18 in the supernatant of cell culture ${ }^{*} p<0.05$, statistically significant difference compared to the group which did not transfect with NLRP3 SiRNA.

and cerebrovascular diseases. The Framingham Heart Study [7] demonstrated that the pathogenesis of atherosclerosis is related to the level of serum HDL-C and ox-LDL. The abnormal lipoprotein metabolism involvement in the pathogenesis and progression of atherosclerosis has multiple complicated mechanisms. Recent studies showed that the NLRP3 inflammasome might be a critical step to bridge abnormal lipoprotein metabolism and atherosclerosis [8, 9]. One study in 2010 [8] found that human macrophages can engulf cholesterol crystals and deposit them in the form of cholesterol esters, and the cholesterol crystals can further induce the release of IL-1 $\beta$ in human mononuclear cells and macrophages in a dose-dependent manner. Moreover, the release of IL- $1 \beta$ is dependent on the activation of caspase-1. This suggests that an NLRP3 inflammasome-mediated signaling pathway is involved in the process. In addition, their study also demonstrated that silencing of the crucial component of the NLRP3 inflammasome completely blocked the cholesterol crystal-induced IL-1 $\beta$ secretion, thus identifying the function of the NLRP3 inflammasome in crystals in macrophages. In 2012, phagocytosis of ox-LDL was shown to induce activation of the NLRP3 inflammasome and secretion of IL- $1 \beta$ in human macrophages. It also showed that ox-LDL upregulates expression of the pro-IL-1 $\beta$ protein, thus priming it for IL-1 $\beta$ secretion. They further suggested that NLRP3 inflammasome activation might be dependent on the generation of reactive oxygen species (ROS) and activation of the cathepsin B signaling pathway [9].

In this study, we used three different lipoproteins - ox-LDL, ox-HDL and HDL - in a mononuclear cell line, Thp-1. Our results showed that ox-LDL and ox-HDL addition not only increases the expression of NLRP3, but also activates the NLRP3 downstream cytokines and caspase- 1 and further induces IL-1 $\beta$ and IL-18 secretion. Moreover, the effects of activation and induction are dose-dependent. Expression of NLRP3 and its downstream inflammatory cytokines is reduced in the presence of HDL. Furthermore, our data demonstrated that NLRP3 siRNA downregulates NLRP3 expression in mononuclear cells, thus leading to a dramatic reduction in the expression of caspase-1, IL-1 $\beta$ and IL-18. The data suggest that activation of the NLRP3 inflammasome is a critical step in caspase- 1 activation and IL-1 $\beta$ and IL-18 secretion. Interference with the NLRP3 inflammasome can significantly inhibit the generation of cytokines, thus impeding the pathogenesis of inflammation.

ox-LDL is formed by the oxidization of LDL, and it is a critical factor in the pathogenesis and progression of atherosclerosis as well as the formation of unstable plaques [10]. The phagocytosis of ox-LDL and its subsequent progression to foam cells are the key steps in the pathogenesis of atherosclerosis. ox-LDL involvement in pathogenesis and progression of atherosclerosis has multiple mechanisms. First, it can induce damage of endothelia, and adhesion and chemotaxis of mononuclear cells to endothelia. In addition, oxLDL can promote vasoconstriction and foam cell formation and affect plaque stability, thus exacerbating atherosclerotic inflammation. A large number of studies have shown that ox-LDL plays a role in atherosclerosis by activating the scavenger receptors of TLR2, TLR4, LOX-1 and others [2, 3,11 ]. This study showed that ox-LDL significantly activates the NLRP3 inflammasome and promotes IL-1 $\beta$ release in inflammation. This suggests that the NLRP3 inflammasome may mediate ox-LDL to 
play a role in atherosclerosis, which is consistent with the study of Jiang et al. [9].

In recent years, our understanding of ox-HDL has gradually increased. A large number of studies suggest that ox-HDL plays a harmful role in the pathogenesis of atherosclerosis, but its mechanism remains unclear. A study [12] proposed that ox-HDL-induced atherosclerosis might be associated with ox-HDL's decreased ability to transport cholesterol, thus leading to the deposition of cholesterol and cholesterol ester on the vessel walls. Another study [13] demonstrated that ox-HDL activates the platelets and promotes the aggregation of platelets and has a cytotoxic effect, thus resulting in the pathogenesis of atherosclerosis. Our study found that ox-HDL can activate the release of the NLRP3 inflammasome and its downstream inflammatory cytokines and caspase-1, and induce the secretion of IL-1 $\beta$ and IL-18. This study is the first to show that ox-HDL might initiate inflammation by activating the NLRP3 inflammasome in the pathogenesis and progression of atherosclerosis.

Decrease in $\mathrm{HDL}-\mathrm{C}$ is a risk index independent of $\operatorname{LDL}-\mathrm{C}[6,14]$. So far, one of the main mechanisms of anti-atherosclerosis for HDL is that HDL mediates the reverse cholesterol transport. HDL transports cholesterol from the peripheral tissues (including atherosclerotic plaques) to the liver for recycling or secretion in the form of cholic acid. This process is referred to as reverse cholesterol transport, which can decrease the deposition of lipids on the vessel walls. Also, HDL can regulate the generation and activity of nitric oxide (NO) in endothelia and improve the vascular endothelial function [15]. Furthermore, HDL can protect vessels via its functions of anti-oxidation, anti-inflammation, anti-thrombosis, promoting fibrinolysis and removing toxic phospholipid $[16,17]$. Our study is the first to demonstrate that HDL addition to mononuclear cells inhibits expression of the NLRP3 inflammasome and its downstream inflammatory cytokines, suggesting that HDL plays an anti-inflammatory role in atherosclerosis by inhibiting the activation of NLRP3 and its downstream cytokines.

In this study, NLRP3 expression was down-regulated in mononuclear cells via NLRP3 siRNA. Down-regulation of NLRP3 remarkably inhibits the secretion of the NLRP3 inflammasome and caspase-1, IL-1 $\beta$ and IL-18 in the presence of ox-LDL, ox-HDL or HDL. This suggests that NLRP3 activation is a critical step in the activation of caspase- 1 and secretion of IL-1 $\beta$ and IL-18. Interference with the NLRP3 inflammasome can considerably inhibit the generation of cytokines, thus impeding the pathogenesis of atherosclerosis. NLRP3 may have a potential to become a therapeutic target that provides a novel perspective for clinical and pharmaceutical research and development.

In our study, we demonstrated that ox-LDL, ox-HDL and HDL are involved in the development of atherosclerosis by regulating activation of the NLRP3 inflammasome and downstream cytokines. However, a clear definition of the underlying mechanism is missing. Some hypotheses about the mechanism of NLRP3 inflammasome activation have been proposed in recent years. Kristiina's study [18] showed that cholesterol crystals induced dose-dependent secretion of mature IL-1 $\beta$ by activating the NLRP3 inflammasome. The crystals were shown to induce leakage of the lysosomal protease cathepsin B into the cytoplasm, and inhibition of this enzyme reduced cholesterol crystal-induced IL-1 $\beta$ secretion, suggesting that NLRP3 inflammasome activation occurred via lysosomal destabilization. It has also been shown that an inhibitor of cathepsin B, CA-074, significantly decreases IL-1 $\beta$ secretion [19]. Thus the lysosome damage and the leakage of lysosomal cysteine protease cathepsin B into the cytoplasm have been inferred to activate the NLRP3 inflammasome [20-22]. Pétrilli's study [23] showed that the NLRP3 inflammasome assembly and caspase-1 recruitment response can be inhibited at high extracellular potassium concentrations in Thp- 1 macrophages, which suggests that potassium efflux may trigger NLRP3 activation. The third mechanism proposed to mediate NLRP3 inflammasome activation involves ROS production [24-27]. This theory proposes that ROS are profusely generated by the stimulation of many NLRP3 activators and the inflammatory responses can be impaired by an ROS inhibitor [28]. As mentioned above, the lipoproteins involved in the development of atherosclerosis by regulating activation of the NLRP3 inflammasome may act through various pathways, among which oxidative stress may be the major contributor. Oxidative stress and lipid oxidation, especially ox-LDL, may release a mass of ROS in the process of atherosclerosis. The ROS promote further oxidation reaction to transform LDL and HDL to ox-LDL and ox-HDL. The vicious circle leads to the continuous secretion of ROS which trigger activation of the NLRP3 inflammasome and downstream cytokines release.

In conclusion, the NLRP3 inflammasome fully participates in the occurrence and progression of atherosclerosis. This study demonstrated that NLRP3 inflammasome mediated signaling regulates ox-LDL-induced inflammation in atherosclerosis. Second, ox-HDL may initiate the inflammation by activating the NLRP3 inflammasome, thus playing a role in the pathogenesis of atherosclerosis. Third, HDL has a protective effect in atherosclerosis by inhibiting the expression of NLRP3 and its downstream cytokines. Finally, in- 
terference with the key components of the NLRP3 inflammasome might become a vital therapeutic target in the treatment of atherosclerosis.

\section{Acknowledgments}

Wang Li Li and Li Gui Hua are the same contributor in the manuscript.

\section{Conflict of interest}

The authors declare no conflict of interest.

\section{References}

1. Galkina E, Ley K. Immune and inflammatory mechanisms of atherosclerosis. Annu Rev Immunol 2009; 27: 165-97.

2. Su X, Ao L, Shi Y. Oxidized low density lipoprotein induces bone morphogenetic protein-2 in coronary artery endothelial cells via Toll-like receptors 2 and 4. J Biol Chem 2011; 286: 12213-20.

3. Mitra S, Goyal T. Oxidized LDL, LOX-1 and atherosclerosis. Cardiovasc Drugs Ther 2011; 25: 419-9.

4. Chavez-Sanchez L, Madrid-Miller A. Activation of TLR2 and TLR4 by minimally modified low-density lipoprotein in human macrophages and monocytes triggers the inflammatory response. Hum Immunol 2010; 71: 737-44.

5. Wang L, QU P. NLRP3 and downstream cytokine expression elevated in the monocytes of patients with coronary artery disease. Arch Med Sci 2014; 10: 791-800.

6. Duewell P, Kono H. NLRP3 inflammasomes are required for atherogenesis and activated by cholesterol crystals. Nature 2010; 464: 1357-61.

7. Franceschini G. Epidemiologic evidence for high density lipoprotein cholesterol as a risk factor for coronary artery disease. Am J Cardiol 2011; 88: 9N-13N.

8. Rajamaki K, Lappalainen J. Cholesterol crystals activate the NLRP3 inflammasome in human macrophages: a novel link between cholesterol metabolism and inflammation. PLoS One 2010; 5: e11765.

9. Jiang $Y$, Wang M. Oxidized low-density lipoprotein induces secretion of interleukin-1beta by macrophages via reactive oxygen species-dependent NLRP3 inflammasome activation. Biochem Biophys Res Commun 2012; 425: 121-6.

10. Burchardt P, Zurawski J. Low-density lipoprotein, its susceptibility to oxidation and the role of lipoprotein-associated phospholipase A2 and carboxyl ester lipase lipases in atherosclerotic plaque formation. Arch Med Sci 2013; 9: 151-8.

11. Xiong YS, YU J. The role of Siglec-1 and SR-BI interaction in the phagocytosis of oxidized low density lipoprotein by macrophages. PLoS One 2013; 8: e58831.

12. Sharma N, Desigan B. The role of oxidized HDL in monocyte/macrophage functions in the pathogenesis of atherosclerosis in Rhesus monkeys. Scand J Clin Lab Invest 1999; 59: 215-25.

13. Hurtado I, Fiol C. In vitro oxidised HDL exerts a cytotoxic effect on macrophages. Atherosclerosis 1996; 125: 39-46.

14. Dodani S, Dong L. Carotid intima media thickness and low high-density lipoprotein (HDL) in South Asian immigrants: could dysfunctional HDL be the missing link? Arch Med Sci 2014; 10: 870-9.

15. Shaul PW. Endothelial nitric oxide synthase, caveolae and the development of atherosclerosis. J Physiol 2003; 547: 21-33.
16. Ansell BJ, Navab M. Inflammatory/antiinflammatory properties of high-density lipoprotein distinguish patients from control subjects better than high-density lipoprotein cholesterol levels and are favorably affected by simvastatin treatment. Circulation 2003; 108: 2751-6.

17. Park SH, Park JH. Involvement of transcription factors in plasma HDL protection against TNF-alpha-induced vascular cell adhesion molecule-1 expression. Int J Biochem Cell Biol 2003; 35: 168-82.

18. Kristiina R, Jani $L$. Cholesterol crystals activate the NLRP3 inflammasome in human macrophages: a novel link between cholesterol metabolism and inflammation. PLoS One 2010; 5: e11765.

19. Buttle DJ, Murata M. CA074 methyl ester: a proinhibitor for intracellular cathepsin B. Arch Biochem Biophys 1992; 299: 377-80.

20. Halle A, Hornung V. The NALP3 inflammasome is involved in the innate immune response to amyloid-beta. Nat Immunol 2008; 9: 857-65.

21. Hornung V, Bauernfeind F. Silica crystals and aluminum salts activate the NALP3 inflammasome through phagosomal destabilization. Nat Immunol 2008; 9: 847-56.

22. Tsai WH, Huang DY. Dual roles of NOD2 in TLR4-mediated signal transduction and -induced inflammatory gene expression in macrophages. Cell Microbiol 2008; 13: 717-30.

23. Petrilli V, Papin S. Activation of the NALP3 inflammasome is triggered by low intracellular potassium concentration. Cell Death Differ 2007; 14: 1583-9.

24. Rubartelli A. Redox control of NLRP3 inflammasome activation in health and disease. J Leukoc Biol 2012; 92: 951-8.

25. Zhou R, Yazdi AS. A role for mitochondria in NLRP3 inflammasome activation. Nature 2011; 469: 221-5.

26. Segovia J, Sabbah A. TLR2/MyD88/NF-kappaB pathway, reactive oxygen species, potassium efflux activates NLRP3/ASC inflammasome during respiratory syncytial virus infection. PLoS One 2012; 7: e29695.

27. Jin C, Frayssinet P. NLRP3 inflammasome plays a critical role in the pathogenesis of hydroxyapatite-associated arthropathy. Proc Natl Acad Sci 2011; 108: 14867-72.

28. Dostert C, Petrilli V. Innate immune activation through Nalp3 inflammasome sensing of asbestos and silica. Science 2008; 320: 674-7. 